\title{
CYCLIC VECTORS AND PARTS OF THE SPECTRUM OF A WEIGHTED SHIFT
}

\author{
BY \\ RALPH GELLAR( ${ }^{(1)}$
}

Introduction. Given a Schauder basis $\left\{y_{n}\right\}_{-\infty}^{\infty}$ in a complex Banach space $\boldsymbol{B}$, with $\left\|y_{n}\right\|=1$ for all $n$, let $a=\left\{a_{n}\right\}_{-\infty}^{\infty}$ be a sequence of nonzero scalars such that the linear operator $T=T_{a}$ defined by $T \sum c_{n} y_{n}=\sum c_{n} a_{n+1} y_{n+1}$ is bounded. The present paper is mostly concerned with the parts of the spectrum, cyclic vectors and invariant subspaces of $T$. It is a continuation in every respect (including numbering of theorems and sections) of the author's paper [11], which was about operators commuting with $T$.

The main source on weighted shift operators are the papers [5], [12], [16], [18][20] of N. K. Nikol'skii. A good deal of information is also scattered throughout Halmos' Hilbert Space Problem Book [13]. For unweighted shifts the best reference is Helson's book [3].

In $\$ \$ 8$ and 14 we describe the parts of the spectrum of $T$ in complete detail. Taylor [2, p. 235] divides the spectrum of a bounded Banach space operator into six parts. Five of these parts may appear in shift operators, but no more than 3 in any one operator.

$\$ 9$ constructs all invariant subspaces of $T$ of finite dimension or codimension.

In [11] we noted that an operator $S$ commuting with $T$ is completely determined by the element $S y_{0} . \S 10$ considers the outcome of assuming (A) that each element $f \in \boldsymbol{B}$ is of the form $S y_{0}$ for some commuting operator $S$. In particular we find all (doubly) cyclic vectors of $T$ under this condition. In $\$ 11$ we construct explicitly the ring of operators commuting with $T$ when $\left\{y_{n}\right\}$ is the usual basis in $c_{0}$ or $l_{1}$ and find n.a.s.c. for hypotheses $(\mathrm{A})$ in these cases.

In $\$ 12$ we give sufficient conditions for (A) for one-sided bases which leads in $\S 13$ to the complete description of the invariant subspaces of certain $T$. Also in $\$ 13$ we find the smallest invariant (or doubly invariant) subspace of $T$ containing an element $f$ whose coefficients decrease sufficiently rapidly.

Preliminary Discussion. A weighted shift is equivalent to an unweighted shift on a nonnormal basis. In [11] we defined a new Schauder basis $\left\{z^{n}\right\}_{-\infty}^{\infty}$ for $\boldsymbol{B}$, such

Presented in part to the Society, April 12, 1968, under the title Weighted shifts. II: Cyclic vectors of the backward shift; received by the editors March 24, 1969.

( $\left.{ }^{1}\right)$ This paper includes both part of the author's dissertation [17] written under Professor Lorch at Columbia University, and research supported by NSF Grant GU2582. 
that $T z^{n}=z^{n+1}$ (generally with $\left\|z^{n}\right\| \neq 1$ ). For each $f \in B, f=\sum_{-\infty}^{\infty} b_{n} z^{n}={ }_{\text {der. }} f(z)$, and we have $T f(z)=z f(z)$.

This heuristic expression of members of $B$ as "Laurent series" suggests a multiplicative and an analytic structure, both of which in fact do have significant relationships to the operator $T$. For example, let $\mathscr{F}=\{f(z) \in \boldsymbol{B} \mid f(z) g(z) \in \boldsymbol{B}$, for all $g(z) \in \boldsymbol{B}\}$. A bounded linear operator commutes with $T$ iff it is of the form $f(T): g(z) \rightarrow f(z) g(z)$ for some $f(z) \in \mathscr{F}[11$, Theorems 1 and 2].

For convenience we reproduce here from [11]:

(2.1) There exists $M>0$ such that if $f(z)=\sum_{-\infty}^{\infty} b_{n} z^{n} \in B$ then

$$
\left|b_{n}\right| \leqq M\|f\| /\left\|z^{n}\right\| \text { for all } n \text {. }
$$

The following result is used implicitly in this paper: If the Laurent series $\sum_{-\infty}^{\infty} b_{n} z^{n}$ and $\sum_{-\infty}^{\infty} c_{n} z^{n}$ both converge to analytic functions on a common annulus, then their formal product $\sum_{n=-\infty}^{\infty}\left(\sum_{j=-\infty}^{\infty} b_{j} c_{n-j}\right) z^{n}$ converges on that annulus to the product of those functions.

\section{Analytic behavior in $B$.}

DEFINITION. Let

$$
\begin{array}{ll}
I^{+}=\liminf _{n \rightarrow \infty}\left\|z^{n}\right\|^{1 / n}, & Q^{+}=\limsup _{n \rightarrow \infty}\left\|z^{n}\right\|^{1 / n}, \\
I^{-}=\limsup _{n \rightarrow-\infty}\left\|z^{n}\right\|^{1 / n}, \quad \text { and } & Q^{-}=\liminf _{n \rightarrow-\infty}\left\|z^{n}\right\|^{1 / n} .
\end{array}
$$

We have $R_{2} \leqq I^{+} \leqq Q^{+} \leqq R_{1}$ and $R_{2} \leqq Q^{-} \leqq I^{-} \leqq R_{1}$.

THEOREM 11. If $f \in \boldsymbol{B}$ then

(1) $f^{+}(z)$ converges to an analytic function in the region $|z|<I^{+}$.

(2) $f^{-}(z)$ converges to an analytic function in the region $|z|>I^{-}$.

(3) If $\mathrm{I}^{-}<I^{+}$, then convergence in $B$ implies uniform convergence of the associated analytic functions on compact subsets of the region $I^{-}<|z|<I^{+}$.

Proof. Let $f(z)=\sum b_{n} z^{n}$. (1) According to (2.1), for $n>0$, we have

Thus

$$
\left|b_{n}\right| \leqq M\|f\| /\left\|z^{n}\right\| \text {. }
$$

$$
\limsup _{n \rightarrow \infty}\left|b_{n}\right|^{1 / n} \leqq \limsup _{n \rightarrow \infty}\left\|z^{n}\right\|^{-1 / n}=\left(\liminf _{n \rightarrow \infty}\left\|z^{n}\right\|^{1 / n}\right)^{-1}=\left(I^{+}\right)^{-1} .
$$

(2) Is proven similarly, using (2.1) for $n<0$.

(3) Given $s$ and $t$ with $I^{-}<s<t<I^{+}$, it suffices to show the existence of a constant $K$ such that if $s \leqq|\lambda| \leqq t$, then $|f(\lambda)| \leqq\|f\| K$ for all $f$ in $B$. Now

$$
|f(\lambda)| \leqq \sum\left|b_{n}\right||\lambda|^{n}=\sum\left\|b_{n} z^{n}\right\| \frac{\left|\lambda^{n}\right|}{\left\|z^{n}\right\|} \leqq\|f\| M\left(\sum_{n=0}^{\infty} \frac{t^{n}}{\left\|z^{n}\right\|}+\sum_{n=-\infty}^{-1} \frac{s^{n}}{\left\|z^{n}\right\|}\right),
$$

where we use (2.1) at the last inequality, and the convergence of the final sums follows from the definition of $I^{+}$and $I^{-}$.

Note that $f \in \boldsymbol{B}$ iff $f^{+} \in \boldsymbol{B}$ and $f^{-} \in \boldsymbol{B}$. 
THEOREM 12. (1) If $f^{+}(z)$ converges to a function analytic on a neighborhood of the set $|z| \leqq Q^{+}$, then $f^{+}(z) \in B$.

(2) If $f^{-}(z)$ converges to a function analytic on a neighborhood of the set $|z| \geqq Q^{-}$, then $f^{-}(z) \in B$.

Proof. (1) The hypothesis implies that $\sum_{n=0}^{\infty}\left|b_{n}\right| t^{n}<\infty$ for some $t>Q^{+}$. By the definition of $Q^{+}$there is a constant $K$ such that $\left\|z^{n}\right\| / t^{n}<K$ for all $n \geqq 0$. It follows that the series for $f^{+}$converges absolutely in $B$ and we have $\left\|f^{+}(z)\right\| \leqq \sum_{0}^{\infty}\left|b_{n}\right|\left\|z^{n}\right\|$ $\leqq K \sum_{0}^{\infty}\left|b_{n}\right| t^{n}$. The proof of (2) is similar.

It is easy to check that not every power series $f^{+}(z)$ with radius of convergence equal to $Q^{+}$lies in $B$.

THEOREM 13. (1) If $f(z)=f^{+}(z) \in B$ and $r_{f}$, the radius of convergence of $f(z)$ satisfies $r_{f}<Q^{+}$, then for each $J$ with $r_{f}<J<Q^{+}$one can write $f(z)=f_{1}(z)+f_{2}(z)$, where the power series $f_{1}(z)$ has Hadamard gaps and the radius of convergence of the power series $f_{2}(z)$ is not less than $J$.

(2) A similar proposition holds when $f(z)=f^{-}(z)$, with $Q^{+}$replaced by $Q^{-}$.

Proof. Pick $K$ such that $I^{+} \leqq r_{f}<J<K<Q^{+}$. (The first inequality is Theorem 11, part (1).) According to the definitions of $I^{+}$and $Q^{+}$, there must exist an infinite set of positive integers $m$ and corresponding integers $m^{\prime}>m$ such that

$$
\left\|z^{m}\right\| \leqq J^{m}, \quad J^{m+j}<\left\|z^{m+j}\right\|<K^{m+j} \text { for } m<m+j<m^{\prime},
$$

and

$$
\left\|z^{m^{\prime}}\right\| \geqq K^{m^{\prime}} \text {. }
$$

We now estimate the minimum value of $m^{\prime}-m$. We have

$$
\|T\|^{m^{\prime}-m} \geqq\left\|z^{m^{\prime}}\right\| /\left\|z^{m}\right\| \geqq(K / J)^{m} .
$$

Thus $m^{\prime}-m \geqq(\log (K / J) / \log \|T\|) m=\theta m$. Let $M_{J}=\left\{n:\left\|z^{n}\right\| \leqq J^{n}\right\}$. If $m$ and $m^{\prime}$ satisfy $\left(^{*}\right)$ and $j<\theta m$, then $m+j \notin M_{J}$. If $f(z)=\sum_{0}^{\infty} b_{n} z^{n}$, define $f_{1}(z)=\sum_{n \in M_{J}} b_{n} z^{n}$ and $f_{2}(z)=\sum_{n \in M}, b_{n} z^{n}$. Now $f_{1}(z)$ clearly has Hadamard gaps in its power series. A proof similar to that in Theorem 11 will show that the radius of convergence of $f_{2}$ is not less than $J$.

8. Some analysis of the spectrum of $T$. We shall be concerned in this section with propositions $\mathscr{P}_{\lambda}$ about shift operators $T$, depending on a complex parameter $\lambda$, which are (1) true for all shift operators when $r_{1}<|\lambda|<r_{2}$ (where $r_{1}$ and $r_{2}$ will be functions of $a)$, (2) true for some shift operators whenever and only whenever $r_{1} \leqq|\lambda| \leqq r_{2}$ and $\lambda \neq 0$, and (3) false for some shift operators when $0 \neq|\lambda|=r_{1} \leqq r_{2}$ or when $r_{1} \leqq r_{2}=|\lambda| \neq 0$. We will say "the situation is ambiguous on the boundary" to describe the situation above. The construction of examples in the ambiguous case will not be given.

Definition. If $f(z)$ is a rational function with no poles at zero or infinity let $f(z)_{+}$and $f(z)_{-}$be its Laurent expansions converging inside a zero-centered circle and outside a zero-centered circle respectively. 
THEOREM 14. (1) $\lambda$ is in the point spectrum of $T$ iff $(\lambda-z)_{+}^{-1}-(\lambda-z)_{-}^{-1} \in \boldsymbol{B}$.

(2) Condition (1) holds if $Q^{+}<|\lambda|<Q^{-}$and

(3) only if $Q^{+} \leqq|\lambda| \leqq Q^{-}$and $\lambda \neq 0$.

The situation is ambiguous on the boundary.

Proof. (1) Simple calculation shows that as Laurent series $(\lambda-z) f(z)=0$ iff $\lambda \neq 0$ and $f(z)$ is a multiple of $\lambda\left((\lambda-z)_{+}^{-1}-(\lambda-z)_{-}^{-1}\right)=\sum_{-\infty}^{\infty} \lambda^{-n} z^{n}$.

(2) When $Q^{+}<|\lambda|<Q^{-}$, Theorem 12 shows that $(\lambda-z)_{+}^{-1}-(\lambda-z)_{-}^{-1}$ lies in $\boldsymbol{B}$.

(3) If $f(z)=\sum_{-\infty}^{\infty} \lambda^{-n} z^{n} \in B$, then by (2.1), $\left|\lambda^{-n}\right| \leqq M\|f\|\left\|z^{n}\right\|^{-1}$ for all $n$. For $n>0,|\lambda| \geqq(M\|f\|)^{-1 / n}\left\|z^{n}\right\|^{1 / n}$ so $|\lambda| \geqq Q^{+}$. For $n<0,|\lambda| \leqq(M\|f\|)^{-1 / n}\left\|z^{n}\right\|^{1 / n}$ so $|\lambda| \leqq Q^{-}$.

NoTE. For $\left\{y_{n}\right\}_{-\infty}^{\infty}$ an orthonormal basis in Hilbert space these facts are found in $[12$, p. 188].

THEOREM 15. (1) $\mathscr{C} \ell((T-\lambda) B) \neq B$ iff the mapping $\mathscr{E}_{\lambda}: \sum b_{n} z^{n} \rightarrow \sum b_{n} \lambda^{n}$ is a bounded linear functional on $\boldsymbol{B}$.

(2) Condition (1) holds if $I^{-}<|\lambda|<I^{+}$and

(3) holds only if $I^{-} \leqq|\lambda| \leqq I^{+}$and $\lambda \neq 0$.

The situation is ambiguous on the boundary.

Proof. (1) Suppose $\mathscr{C} \ell((T-\lambda) \boldsymbol{B}) \neq \boldsymbol{B}$. Then there exists a nonzero bounded linear functional $F$ on $B$ vanishing on $\mathscr{C} \ell((T-\lambda) B)$. We have $F(z-\lambda) z^{n}=0$ for all $n$. Thus $F z^{n+1}=\lambda F z^{n}$ so $F$ is a scalar multiple of $\mathscr{E}_{\lambda}$. Conversely, if $\mathscr{E}_{\lambda}$ is continuous it vanishes on $\mathscr{C} \ell((T-\lambda) B)$, but $\mathscr{E}_{\lambda} \neq 0$.

(2) The continuity of $\mathscr{E}_{\lambda}$ for $I^{-}<|\lambda|<I^{+}$follows immediately from Theorem 11 part (3).

(3) Suppose $\left\|\mathscr{E}_{\lambda}\right\| \leqq M$. Then $\left|\lambda^{n}\right| \leqq M\left\|z^{n}\right\|$ for all $n$. For $n>0,|\lambda| \leqq M^{1 / n}\left\|z^{n}\right\|^{1 / n}$ so $|\lambda| \leqq I^{+}$. For $n<0,|\lambda| \geqq M^{1 / n}\|z\|^{1 / n}$ so $|\lambda| \geqq I^{-}$. When $\lambda=0, \mathscr{C} \ell((T-\lambda) B)=B$ and $\mathscr{E}_{\lambda}$ is not bounded.

We now turn to questions of invariant subspaces and cyclic vectors, but shall return to examine the spectrum in more detail in $\$ 14$.

9. Invariant subspaces of finite dimension and codimension. We use the word subspace to mean a closed linear manifold. We use the unqualified word invariant to describe a set $N \subseteq B$ such that if $f \in N$ then $T f \in N$. We say a set $N$ is doubly invariant if it is invariant and whenever $f \in N$ and $z^{-1} f \in B$ then $z^{-1} f \in N$. We denote by $\boldsymbol{C}_{f}$ and $\boldsymbol{E}_{f}$ respectively, the smallest invariant and the smallest doubly invariant subspace of $B$ containing $f$. We say a subspace $N$ is of codimension $n$ in $\boldsymbol{B}$ if the quotient space $\boldsymbol{M}=\boldsymbol{B} / \boldsymbol{N}$ has dimension $n$. Let $f \rightarrow[f]$ be the natural map from $B$ to $M$. If $N$ is an invariant subspace then the operator $T_{M}$ on $M$ defined by $T_{M}[f]=[z f]$ is bounded.

Lemma 1. (1) Let $N$ be an invariant subspace of finite codimension $n$ in $\boldsymbol{B}$. Among those polynomials satisfying $p(T) B \subseteq N$ there is a minimal monic polynomial $p . p$ is the minimal polynomial of $T_{M}$. Degree $p=n . \mathscr{C} \ell(p(T) B)=N$. 
(2) Let $N$ be an invariant subspace. If $p\left(T_{M}\right)=0$ for some polynomial $p$, then codimension $\boldsymbol{N}$ is finite.

Proof. (1) Note that $p\left(T_{M}\right)=0$ iff $p(T) B \subseteq N$. Thus the existence of the minimal polynomial is assured and it is the minimal polynomial of $T_{M}, p . N_{1}=\mathscr{C} \ell(p(T) B) \subseteq N$ so that the natural maps $B \rightarrow B / N_{1} \rightarrow B / N$ are surjections. Thus the sequence $\left\{\left[z^{n}\right]\right\}_{-\infty}^{\infty}$ is complete in $B / N_{1}$. But $\left[z^{n} p(z)\right]=0$ in $B / N_{1}$ for all $n$. It follows that $\left\{\left[z^{n}\right]\right\}_{0}^{m-1}$ is complete in $B / N_{1}$ where $m$ is the degree of $p$. So dimension $B / N_{1} \leqq m$. But $p$ is the minimal polynomial of $T_{M}$ so $m \leqq n$. It follows that the second map above is an isomorphism, $n=m$ and $N_{1}=N$.

(2) As above, the hypothesis implies that $\left\{\left[z^{n}\right]\right\}_{0}^{m-1}$ is complete in $M$ where $m$ is the degree of $p$, so dimension $\boldsymbol{M}$ is finite.

Theorems 14 and 15 give sufficient information to enable us to describe the invariant subspaces of dimension and codimension 1 . A one-dimensional invariant subspace is constructed by choosing an element of $B$ of the form $(\lambda-z)_{+}^{-1}-(\lambda-z)_{-}^{-1}$ as its basis. This can be done if $Q^{+}<|\lambda|<Q^{-}$, only if $Q^{+} \leqq|\lambda| \leqq Q^{-}$and $\lambda \neq 0$, and the boundary situation is ambiguous.

If $\boldsymbol{N}$ is a one-codimensional invariant subspace, Lemma 1 shows that

$$
\boldsymbol{N}=\mathscr{C} \ell((T-\lambda) B)
$$

for some $\lambda$. Thus a one-codimensional invariant subspace is constructed by choosing $\lambda$ such that $\mathscr{E}_{\lambda}$ is bounded and letting the subspace be the set of elements of $\boldsymbol{B}$ which vanish at $\lambda$.

We shall generalize Theorems 14 and 15. The new proofs are too similar to those theorems' to be included. As corollaries we shall find all finite dimensional and finite codimensional invariant subspaces.

THEOREM 14A. (1) kernel $(T-\lambda)^{n} \notin \operatorname{kernel}(T-\lambda)^{n-1}$ for $n>0$ iff

$$
(\lambda-z)_{+}^{-n}-(\lambda-z)_{-}^{-n} \in B \text {. }
$$

(2) Condition (1) holds for all $n>0$ if $Q^{+}<|\lambda|<Q^{-}$.

(3) Condition (1) holds for no $n>0$ unless $Q^{+} \leqq|\lambda| \leqq Q^{-}$and $\lambda \neq 0$.

(4) Ambiguity on the boundary: For each $\lambda$ "on the boundary" there exists $k_{\lambda} a$ positive integer, 0 or $\infty$ such that condition (1) holds if $k_{\lambda} \geqq n>0$, and fails to hold if $n>k_{\lambda}$. (The value of $k_{\lambda}$ is "ambiguous".)

THEOREM 15A. (1) $\mathscr{C} \ell\left((T-\lambda)^{n} B\right) \notin \mathscr{C} \ell\left((T-\lambda)^{n-1} B\right)$ for $n>0$ iff the mapping $f(z) \rightarrow \mathscr{E}_{\lambda}\left(D^{n-1} f(z)\right)$, which evaluates at $\lambda$ the $(n-1)$ st formal derivative of each $f(z) \in \boldsymbol{B}$, is a bounded linear functional on $\boldsymbol{B}$.

(2) Condition (1) holds for all $n>0$ if $I^{-}<|\lambda|<I^{+}$and

(3) holds for no $n>0$ unless $I^{-} \leqq|\lambda| \leqq I^{+}$and $\lambda \neq 0$.

(4) Ambiguity on the boundary: For each $\lambda$ "on the boundary" there exists $j_{\lambda}$ a positive integer, 0 or $\infty$ such that condition (1) holds if $j_{\lambda} \geqq n>0$, and fails to hold if $n>j_{\lambda}$. 
We construct any finite dimensional invariant subspace $N$ by choosing points $\lambda_{i}, i=1, \ldots, j$, satisfying $Q^{+} \leqq\left|\lambda_{i}\right| \leqq Q^{-}$and $\lambda_{i} \neq 0$, and choosing positive integers $m_{i}$, with $m_{i} \leqq k_{\lambda_{i}}$ if $\lambda_{i}$ is "on the boundary". Let $N$ be the subspace with basis $\left\{\left(\lambda_{i}-z\right)_{+}^{-n}-\left(\lambda_{i}-z\right)_{-}^{-n} \mid i=1, \ldots, j, 1 \leqq n \leqq m_{i}\right\}$. Since

$$
(\lambda-T)\left((\lambda-z)_{+}^{-n}-(\lambda-z)_{-}^{-n}\right)=(\lambda-z)_{+}^{-n+1}-(\lambda-z)_{-}^{-n+1}
$$

if $n>1$, or $=0$ if $n=1$, it is clear that $N$ is invariant. That every finite dimensional invariant subspace must be of this form follows easily from Theorem 14A and the well known structure of operators in finite dimensional spaces.

Dimension $C_{f}$ is finite iff $f=(g(z))_{+}-(g(z))_{-}$for some rational function $g(z)$. The formal equation $z^{-1} f=z^{-1}(1 / \lambda)(\lambda-z) f+(1 / \lambda) f$ shows that every finite dimensional invariant subspace is doubly invariant.

We construct any finite codimensional subspace $N$ by choosing points $\lambda_{i}, i=1, \ldots, j$ satisfying $I^{-} \leqq\left|\lambda_{i}\right| \leqq I^{+}$and $\lambda_{i} \neq 0$, and choosing positive integers $m_{i}$, with $m_{i} \leqq j_{\lambda_{i}}$ if $\lambda_{i}$ is "on the boundary". Let $N$ be the set of $f(z) \in B$ which vanish to degree at least $m_{i}$ at each $\lambda_{i}$ (that is, $\mathscr{E}_{\lambda}\left(D^{n} f(z)\right)=0$ if $\lambda=\lambda_{i}$ and $0 \leqq n<m_{i}$ ). Codimension $N$ is $\sum_{i=1}^{j} m_{i} . \prod_{i=1}^{j}\left(z-\lambda_{i}\right)^{m_{i}}$ is the minimal polynomial of $T_{M}$. It is clear that any $N$ chosen as above is not only an invariant subspace but is doubly invariant also.

Conversely if $\boldsymbol{N}$ is invariant of finite codimension, the minimal polynomial $p$ of $T_{M}$ can be factored as above. It is an easy consequence of Theorem 15A that $\lambda_{i}$ and $m_{i}$ must satisfy the restrictions listed above. By Lemma $1, N=\mathscr{C} \ell(p(T) B)$ so $N$ is included in the examples above.

10. When $\boldsymbol{B}$ is an algebra. As sets of power series, $\mathscr{F} \subseteq B$. Throughout this section we shall investigate the case where $\mathscr{F}=\boldsymbol{B}$. Theorems 1 and 2 show that $\mathscr{F}=\boldsymbol{B}$ iff $f(z) \in \boldsymbol{B}$ and $g(z) \in \boldsymbol{B}$ imply that $f(z) g(z) \in \boldsymbol{B}$.

Note 1. The natural map from $\mathscr{F}$ to $B$ which sends $f(z)$ to $f(z)$ is norm-decreasing: $\|f(z)\|_{B}=\|f(T) 1\|_{B} \leqq\|f(T)\|=\|f(z)\|_{F}$. If $\mathscr{F}=B$ this map (which preserves products) is $1-1$ and onto so $\mathscr{F}$ and $B$ are topalgebraically isomorphic. Thus we can transfer ideal theory from $\mathscr{F}$ to $B$ and $\left\{z^{n}\right\}_{-\infty}^{\infty}$ is a Schauder basis in $\mathscr{F}$.

TheOREM 16. Suppose $\mathscr{F}=B$. Then (1) spectrum $T=D=\left\{z\left|R_{2} \leqq\right| z \mid \leqq R_{1}\right\}$. $0<R_{2}=Q^{-}=I^{-} \leqq I^{+}=Q^{+}=R_{1}$.

(2) $j_{\lambda} \geqq 1$ for each point $\lambda$ "on the boundary". There is one maximal ideal of $\boldsymbol{B}$ corresponding to each point $\lambda$ of $D$, and the associated multiplicative functional is $\mathscr{E}_{\lambda}$.

(3) If $f(z)=\sum b_{n} z^{n} \in \boldsymbol{B}$, then the Laurent series $\sum b_{n} z^{n}$ converges uniformly (as a function) on $D$ to the function $f(z)$ (which must then be continuous on $D$ and analytic in the interior of $D$ ).

Proof. Let $N$ be a maximal ideal in $\mathscr{F}(=B)$ and let $F$ be the associated multiplicative linear functional. $z-\lambda \in N$ for a unique complex $\lambda$, so $F(z-\lambda)=0$. Then $F\left(z^{n}(z-\lambda)\right)=0$ for all $n$. Thus $F\left(z^{n+1}\right)=\lambda F\left(z^{n}\right)$, and we also know that $F(1)=1$, so $F$ is $\mathscr{E}_{\lambda}$. By Theorem 15, part (3), we must have $I^{-} \leqq|\lambda| \leqq I^{+}$and $\lambda \neq 0$. 
Now suppose $\lambda \in$ spectrum $T$. Then there exists a maximal ideal $N$ in $\mathscr{F}(=B)$ such that $z-\lambda \in N$. Thus spectrum $T \subseteq\left\{\lambda\left|I^{-} \leqq\right| \lambda \mid \leqq I^{+}\right.$and $\left.\lambda \neq 0\right\}$. But by Theorem 6, $D \subseteq$ spectrum $T$. (1) and (2) follow immediately.

The multiplicative functionals $\mathscr{E}_{\lambda}$ are all of norm 1 on $\mathscr{F}$ and so are uniformly bounded on $\boldsymbol{B}$ for all $\lambda \in D$. Thus convergence in $\boldsymbol{B}$ implies uniform convergence on $D$. Since for each $f(z)=\sum b_{n} z^{n} \in \boldsymbol{B}$, the series converges in $\boldsymbol{B}$ to $f(z)$, part (3) follows.

THEOREM 17. If $\mathscr{F}=\boldsymbol{B}$ every doubly invariant subspace is a closed ideal and vice versa. $E_{f}=B$ iff $f$ has no zeros on $D$.

Proof. Since $T^{-1}$ is bounded, a doubly invariant subspace $N$ is invariant under both $T$ and $T^{-1}$. Pick $f \in N$ and $g=\sum b_{n} z^{n} \in B$. Since $f \in \mathscr{F}, g f=\sum b_{n} z^{n} f \in N$ so $N$ is an ideal. That every ideal is doubly invariant is trivial.

We conclude that $\boldsymbol{E}_{f}=\boldsymbol{B}$ iff $f$ is contained in no proper ideal iff $\mathscr{E}_{\lambda}(f) \neq 0$ for all $\lambda \in D$.

NoTE 2. If we had been working with a one-sided basis $\left\{y_{n}\right\}_{0}^{\infty}$ the statements of the last two theorems would have to be varied slightly. In particular, $\mathscr{E}_{\lambda}$ would be bounded for $\lambda=0$. Also in the above theorem we would replace $E_{f}$ by $C_{f}$, and "doubly invariant" by "invariant".

THEOREM 18. Assume $\mathscr{F}=B$. Suppose $f$ does not vanish on the zero-centered circles of radii $R_{1}$ and $R_{2}$. Then $E_{f}$ is of finite codimension. $E_{f}$ is the set of functions vanishing to at least the same orders at the same points as $f$.

Proof. Let $\boldsymbol{M}=\boldsymbol{B} / \boldsymbol{E}_{f}=\mathscr{F} / \boldsymbol{E}_{f}$. Since $\boldsymbol{E}_{f}$ is a closed ideal, $\boldsymbol{M}$ is topalgebraically isomorphic to a quotient Banach algebra. Each maximal ideal in $M$ is the quotient of a maximal ideal in $\boldsymbol{B}$ containing $\boldsymbol{E}_{f}$, the smallest closed ideal containing $f$. Thus the associated linear functionals to maximal ideals in $\boldsymbol{M}$ are of the form $[g] \rightarrow \mathscr{E}_{\lambda}(g)$ for precisely those $\lambda$ for which $\mathscr{E}_{\lambda}(f)=0$. Using the same arguments as in the proof of Theorem 16, we may conclude that spectrum $T_{M}$ =zeros of $f \subseteq$ interior of $D$.

$f(z)=\sum b_{n} z^{n}$ converges uniformly in some neighborhood of spectrum $T_{M}$ so the usual functional calculus defines $f\left(T_{M}\right)=\sum b_{n} T_{M}^{n}$ where the series converges in the uniform operator topology. Now $f\left(T_{M}\right)[1]=\sum b_{n} T_{M}^{n}[1]=\sum b_{n}\left[z^{n}\right]=[f]=0$. Also, since $f\left(T_{M}\right)$ commutes with $T_{M}, f\left(T_{M}\right)\left[z^{m}\right]=f\left(T_{M}\right) T_{M}^{m}[1]=0$ for all $m$. Lorch [1, Theorems 8 and 9] shows that if under the usual functional calculus, a function of an operator is zero, that operator satisfies a polynomial identity. Part (2) of Lemma 1 then shows that $E_{f}$ is finite codimensional.

According to our description of invariant subspaces of finite codimension, $\boldsymbol{E}_{f}$ must be the set of all elements of $B$ vanishing at certain specific points to at least certain specific orders. But clearly every element of $\boldsymbol{E}_{f}$ vanishes wherever $f$ does, and to at least as high order as $f$ (note that we do not admit the concept of an 
element in $\boldsymbol{B}$ vanishing at $\lambda$ "on the boundary" to any order higher than $j_{\lambda}$ ). On the other hand, $f$ itself vanishes only as $f$ does. Thus $E_{f}$ must be as advertised.

(Note: working with one-sided bases we must replace $\boldsymbol{E}_{f}$ above by $\boldsymbol{C}_{f}$.)

Note: When $B=\mathscr{F}$, the method used in the above proof allows us to find the spectrum of $f\left(T_{M}\right)$ for any (doubly) invariant subspace $N$ and any $f \in \mathscr{F}$. The analogous problem when $B$ is the space $\boldsymbol{H}^{2}$ (and $\mathscr{F}=\boldsymbol{H}^{\infty}$ ) has been solved by $\mathrm{P}$. Fuhrmann [14].

11. Shifts on $c_{0}$ and $l_{1}$. In this section we study "shifts on $c_{0}$ and $l_{1}$ ", that is weighted shift operators on the usual basis of these spaces. The fact that the norm of an operator on $c_{0}$ or $l_{1}$ can be calculated from the coefficients of its matrix makes possible more precise information in this setting.

We use two-sided spaces. We define $c_{0}$ to be the set of sequences $c=\left\{c_{n}\right\}_{-\infty}^{\infty}$ converging to zero in both directions, and with the sup norm. For $l_{1}$ we require $\sum_{-\infty}^{\infty}\left|c_{n}\right|<\infty$ and this sum is the norm.

The transformation $T c=d$ where $d_{i}=\sum_{j=-\infty}^{\infty} a_{i j} c_{j}$ is a bounded linear operator from $c_{0}$ to $c_{0}$ if the matrix $\left(a_{i j}\right)$ satisfies $\lim _{i \rightarrow \infty} a_{i j}=\lim _{i \rightarrow-\infty} a_{i j}=0$ for all $j$, and $\sup _{i} \sum_{j=-\infty}^{\infty}\left|a_{i j}\right|<\infty$, and this last constant is then the norm of the transformation. For $l_{1}$ the requirement is $\sup _{j} \sum_{i=-\infty}^{\infty}\left|a_{i j}\right|<\infty$ and this constant is the norm of $T$. (Cf. [2, p. 217 and p. 220] for similar results for the one-sided spaces.)

The usual basis of $c_{0}$ or $l_{1}$ is $\left\{y_{n}\right\}_{-\infty}^{\infty}$ where $y_{n}$ is the sequence with 1 in the $n$th place, 0 elsewhere. This is an $N$-basis (see Theorem 10 ).

In the remainder of this section we shall assume that $T$ is a shift on $c_{0}$ or on $l_{1}$.

THEOREM 19. In $c_{0}, f(z)=\sum b_{n} z^{n} \in \mathscr{F}$ iff.

$$
\lim _{i \rightarrow \infty}\left|b_{i-j}\right|\left\|z^{i}\right\|=\lim _{i \rightarrow-\infty}=0 \text { and } \sup _{i} \sum_{j=-\infty}^{\infty}\left|b_{i-j}\right| \frac{\left\|z^{i}\right\|}{\left\|z^{j}\right\|}<\infty
$$

and this constant is $\|f(T)\|$. For $l_{1}$, the corresponding requirement and constant is

$$
\|f(T)\|=\sup _{j} \sum_{i=-\infty}^{\infty}\left|b_{i-j}\right| \frac{\left\|z^{i}\right\|}{\left\|z^{j}\right\|}<\infty .
$$

Proof. The matrix of $f(T)$ with respect to the usual basis in $c_{0}$ or in $l_{1}$ satisfies $\left|a_{i j}\right|=\left|b_{i-j}\right|\left\|z^{i}\right\| /\left\|z^{j}\right\|$.

COROLLARY 3. If $f(z)=\sum b_{n} z^{n} \in \mathscr{F}$, then in the strong operator topology

$$
\lim _{k, j \rightarrow \infty} \sum_{n=-k}^{j} b_{n} T^{n}=f(T)
$$

Proof. First, calculation of operator norms by the formulas of Theorem 19 shows that $\left\|\sum_{n=-k}^{j} b_{n} T^{n}\right\| \leqq\|f(T)\|$.

We must show that

$$
\lim _{k, j \rightarrow \infty} \sum_{n=-k}^{j} b_{n} T^{n} g=f(T) g \quad \text { for all } g \in \boldsymbol{B} .
$$


This equation holds for $g=z^{m}$, because $z^{m} f$ lies in $B$ for all $m$, and $\left(^{*}\right)$ simply expresses the fact that the Laurent series for $z^{m} f$ converges to $z^{m} f$ in $B$. Because of the uniform boundedness of the operators proven above, $\left({ }^{*}\right)$ then extends to hold for all elements in the closed span of $\left\{z^{m}\right\}_{-\infty}^{\infty}$.

COROllaRY 4. If $N$ is a doubly invariant subspace and $f \in \mathscr{F}$, then $N$ is invariant under $f(T)\left({ }^{3}\right)$.

THEOREM 20. (1) If $f=\sum b_{n} z^{n} \in \mathscr{F}$ then $f^{+} \in \mathscr{F}$ and $f^{-} \in \mathscr{F}$.

(2) If $f \in \mathscr{F}, f^{+}(z)$ is bounded on the region $|z|<R_{1}$, and $f^{-}(z)$ is bounded on the region $R_{2}<|z|$, both bounded by $\|f(T)\|$.

Proof. (1) is a simple consequence of Theorem 19. When $R_{2}<R_{1},(2)$ is implied by Theorem 5 .

We may assume $\|f(T)\| \leqq 1$. Then by Theorem $19,\left\|f^{+}(T)\right\| \leqq 1$. We have $\left|b_{n}\right| R_{1}^{n} \leqq\left\|b_{n} T^{n}\right\| \leqq\left\|f^{+}(T)\right\| \leqq 1$, for all $n \geqq 0$, where the middle inequality follows from Theorem 19, and the first inequality is derived as follows: By Theorem 10, the spectral radius of $T=R_{1}$. Thus the spectral radius of $T^{n}=R_{1}^{n} \leqq\left\|T^{n}\right\|$.

We calculate $\left|f^{+}(\lambda)\right| \leqq \sum_{0}^{\infty}\left|b_{n}\right||\lambda|^{n}=\sum\left|b_{n}\right| R_{1}^{n}\left(|\lambda| / R_{1}\right)^{n} \leqq\left(1-\left(|\lambda| / R_{1}\right)\right)^{-1}=s_{\lambda}$ for $|\lambda|<R_{1}$. Since we also will have $\left\|\left(f^{+}\right)^{n}(T)\right\| \leqq 1$ for $n>0$, a repetition of the above argument shows that $\left|f^{+}(\lambda)\right|^{n} \leqq s_{\lambda}$ for all $n>0$. We conclude that $\left|f^{+}(\lambda)\right| \leqq 1$.

A similar proof works for $f^{-}$.

We have proved in Theorem 20 that if $R_{1}=R_{2}$ and $f \in \mathscr{F}$, then $f(z)$ is the "Fourier series" of a function bounded and measurable on $|z|=R_{1}$.

COROllaRY 5. If $R_{2}<R_{1}$ or if $R_{2}=0, T$ has no reducing subspaces. Suppose $R_{2}=R_{1}$ and $N$ is a reducing subspace. Then there is a measurable subset $E$ of the circle $|z|=R_{1}$ such that $N$ is the set of $f(z)$ in $B$ vanishing on $E$, in the sense that $\boldsymbol{N}=\{f \in \boldsymbol{B} \mid p(z) f(z)=f(z)\}$ where $p(z)$ is the "Fourier series" of the function which is equal to 0 on $E$, and 1 on the rest of the circle.

Proof. By definition a reducing subspace is the range of a nontrivial projection which commutes with $T$. Thus we have $N=p(T) B$ and $(p(T))^{2}=p(T)$. Corollary 2 , in $\S 3$ shows this can only happen if $0 \neq R_{1}=R_{2}$. In this case we have $(p(z))^{2}=p(z)$ as formal series, so $p(z)$ is the "Fourier series" of a measurable function taking on only the values 0 and 1 . The corollary follows without difficulty $\left({ }^{4}\right)$.

( ${ }^{3}$ ) Added in Proof. In The commutants of certain Hilbert space operators, (to appear) A. L. Shields and L. J. Wallen have proven that any operator commuting with a weighted shift on the one-sided 2 space is a weak limit of polynomials in the shift. Therefore Corollary 4 holds for these shifts also.

( $\left.{ }^{4}\right)$ Added in Proof. A weighted shift on $c_{0}$ or $l_{1}$ has no nontrivial reducing subspaces. The author is grateful to $M$. Hilden for the following argument: For convenience assume $R_{1}=1$. Suppose $p(z)=\sum_{-\infty}^{\infty} b_{n} z^{n}$, and let $f\left(e^{i \theta}\right)=\sum_{0}^{\infty} b_{n} e^{i n \theta}$. Re $f\left(e^{i \theta}\right)=p\left(e^{i \theta}\right)=$ characteristic function of $E$. By Theorem 20, $f\left(e^{i \theta}\right) \in H^{\infty}$. Then $g\left(e^{i \theta}\right)=e^{2 \pi i f\left(e^{i \theta}\right)} \in H^{\infty}$. Im $g\left(e^{i \theta}\right)=0$ a.e. Since a function in $H^{\infty}$ is determined within a constant by its imaginary part, $g\left(e^{i \theta}\right), f\left(e^{i \theta}\right)$ and $p\left(e^{i \theta}\right)$ are all constant. 
The above proposition is true (and well known) for the unweighted shift on an orthonormal basis in Hilbert space. Helson [3, p. 7] attributes it to Wiener. Halmos $[4$, p. 493] seems a bit doubtful about that.

THEOREM 21. $\boldsymbol{B}=\mathscr{F}$ iff

$$
\sup _{i} \sum_{j=-\infty}^{\infty} \frac{\left\|z^{i}\right\|}{\left\|z^{j}\right\|\left\|z^{i-j}\right\|}<\infty \quad\left(\text { for } c_{0}\right)
$$

and iff

$$
\sup _{i, j} \frac{\left\|z^{i+j}\right\|}{\left\|z^{i}\right\|\left\|z^{j}\right\|}<\infty \quad\left(\text { for } l_{1}\right) .
$$

Proof. Suppose $B=\mathscr{F}$. Then Note 1 of $\S 10$ implies that there exists a constant $K$ such that $\|f(T)\| \leqq K\|f\|$ for all $f \in B$. In $c_{0}: f(z)=\sum_{n=-m}^{m}\left\|z^{n}\right\|^{-1} z^{n}$ has $B$-norm 1 . Then

$$
K \geqq\|f(T)\|=\sup _{i} \sum_{j=i-m}^{i+m} \frac{\left\|z^{i}\right\|}{\left\|z^{i-j}\right\|\left\|z^{j}\right\|},
$$

using Theorem 19. In $l_{1}: f(z)=\left\|z^{m}\right\|^{-1} z^{m}$ does the trick.

Conversely: If $f(z) \in \boldsymbol{B}$, then in $c_{0}$,

$$
\|f(T)\|=\sup _{i} \sum_{j=-\infty}^{\infty}\left|b_{i-j}\right| \frac{\left\|z^{i}\right\|}{\left\|z^{j}\right\|} \leqq\|f\| \sup _{i} \sum_{j=-\infty}^{\infty} \frac{\left\|z^{i}\right\|}{\left\|z^{i-j}\right\|\left\|z^{j}\right\|},
$$

where we used (2.1) at the last step. We must also check that $z^{n} f \in B$ for all $n$ and all $f \in \boldsymbol{B}$. This follows since

$$
\sup _{i} \frac{\left\|z^{i}\right\|}{\left\|z^{i+1}\right\|\left\|z^{-1}\right\|}<\infty
$$

which implies $R_{2}>0$, and then $T^{-1}$ is bounded by Theorem 10 .

In $l_{1}$,

$$
\begin{aligned}
\|f(T)\| & =\sup _{j} \sum_{i=-\infty}^{\infty}\left|b_{i-j}\right| \frac{\left\|z^{i}\right\|}{\left\|z^{j}\right\|} \\
& =\sup _{j} \sum_{i=-\infty}^{\infty}\left|b_{i-j}\right|\left\|z^{i-j}\right\|\left(\frac{\left\|z^{i}\right\|}{\left\|z^{i-j}\right\|\left\|z^{j}\right\|}\right) \leqq\|f\| \sup _{i, j} \frac{\left\|z^{i}\right\|}{\left\|z^{i-j}\right\|\left\|z^{j}\right\|},
\end{aligned}
$$

since $\sum_{i--\infty}^{\infty}\left|b_{i-j}\right|\left\|z^{i-j}\right\|=\|f\|$.

12. One-sided bases. Suppose throughout this section that we had started with a one-sided basis $\left\{y_{n}\right\}_{0}^{\infty}$ and the sequence $a=\left\{a_{n}\right\}_{1}^{\infty}$ to determine a shift operator $T$. We derive conditions guaranteeing $B=\mathscr{F}$. This will lead, in $\S 13$, to theorems concerning invariant subspaces.

THEOREM 22. If there exists $m>0$ such that

$$
\sum_{k=2 m}^{\infty} \sum_{i=m}^{k-m} \frac{\left\|z^{k}\right\|}{\left\|z^{i}\right\|\left\|z^{k-i}\right\|}<\infty
$$

then $B=\mathscr{F}$ and $I^{+}=0$. 
Proof. Let $f=\sum_{0}^{\infty} b_{n} z^{n}$ and $g=\sum_{0}^{\infty} c_{n} z^{n}$ lie in $B$. Then $f=p+u$ and $g=q+v$ where $p=\sum_{0}^{m-1} b_{n} z^{n}$ and $q=\sum_{0}^{m-1} c_{n} z^{n}$. The product $u(z) v(z)$ lies in $\boldsymbol{B}$. In fact

$$
\|u v\| \leqq \sum_{k=2 m}^{\infty} \sum_{i=m}^{k-m}\left\|b_{i} c_{k-i} z^{k}\right\| \leqq M^{2}\|u\|\|v\| \sum_{k=2 m}^{\infty} \sum_{i=m}^{k-m} \frac{\left\|z^{k}\right\|}{\left\|z^{i}\right\|\left\|z^{k-i}\right\|}<\infty,
$$

where we use (2.1) at the middle inequality. Then $f g=p q+p v+u q+u v$ lies in $\boldsymbol{B}$. $\boldsymbol{B}=\mathscr{F}$. To show $I^{+}=0$ note that we have

$$
\sum_{k=2 m}^{\infty} \frac{\left\|z^{k}\right\|}{\left\|z^{k-m}\right\|}<\infty
$$

Thus $\lim _{k}\left\|z^{k}\right\| /\left\|z^{k-m}\right\|=0$ and $\lim _{n \rightarrow \infty}\left\|z^{n}\right\|^{1 / n}=0$.

THEOREM 23. Suppose $\left\{y_{m}\right\}_{0}^{\infty}$ is an $N$-basis (see Theorem 10) and $\left\|T_{1}\right\|=1$. Then if there is an $m>0$ such that

$$
\sum_{j=m}^{\infty} \sup _{i \geq m} \frac{\left\|z^{i+j}\right\|}{\left\|z^{i}\right\|\left\|z^{j}\right\|}<\infty
$$

$\boldsymbol{B}=\mathscr{F}$ and $I^{+}=0$.

Proof. As in Theorem 22 we let $f=p+u$ and $g=q+v$ and show $u v$ lies in $\boldsymbol{B}$.

Note that $\left\{y_{n}\right\}_{m}^{\infty}$ is also an $N$-basis for the space it spans and that $T_{1}$ restricted to this space has norm 1 also. Using (6.1) and (2.1) we obtain

$$
\begin{aligned}
\|u v\| \leqq \sum_{j=m}^{\infty}\left\|u c_{j} z^{j}\right\| & \leqq N M\|u\|\|v\| \sum_{j=m}^{\infty} \frac{\sup _{i \leq m} \prod_{k=i+1}^{i+j}\left|a_{k}\right|}{\left\|z^{j}\right\|} \\
& =N M\|u\|\|v\| \sum_{j=m}^{\infty} \sup _{i \geq m} \frac{\left\|z^{i+j}\right\|}{\left\|z^{i}\right\|\left\|z^{j}\right\|}<\infty .
\end{aligned}
$$

The proof that $I^{+}=0$ is the same as in Theorem 22 .

13. Invariant subspaces. If $T$ is a shift with weights $a=\left\{a_{n}\right\}_{1}^{\infty}$ on a one-sided basis $\left\{y_{n}\right\}_{0}^{\infty}$ then for $n \geqq 0, B_{n}=\mathscr{C} \ell\left(T^{n} B\right)$ is an invariant subspace of $T$. $\left\{y_{i}\right\}_{n}^{\infty}$ is a basis for $\boldsymbol{B}_{n} . T$ restricted to $\boldsymbol{B}_{n}$ is a shift operator on that basis with weights $\left\{a_{i}\right\}_{n+1}^{\infty}$.

THEOREM 24. If for each $n>0, B_{n}$ (considered on its own as the domain of the shift operator $T$ ) is an algebra (as described in $\$ 10$ ), and if in addition $I^{+}=0$, then the only invariant subspaces of $T$ in $B$ are the subspaces $B_{n}$.

Proof. First note that for the power series representations of all $\boldsymbol{B}_{n}$, we have $I^{+}=0$ also.

Let $\boldsymbol{N}$ be an invariant subspace. Let $n$ be the largest integer such that $N \subseteq B_{n}$. Choose $f \in N$ with $f \in \boldsymbol{B}_{n} \mid \boldsymbol{B}_{n+1}$. In the power series representation of $\boldsymbol{B}_{n},(f)_{0}=$ 
$\mathscr{E}_{0}(f) \neq 0$. By Theorem 17 and the comment after it $C_{f}=B_{n}$. Since $C_{f} \subset N$ we are done.

COROLlARY 6. For a one-sided shift the only invariant subspaces are of the form $\boldsymbol{B}_{n}$ if any of the following conditions hold:

(a) For each $n \geqq 0$, there is an $m>0$ such that

$$
\sum_{k=2 m}^{\infty} \sum_{i=m}^{k-m}\left|\frac{a_{n+i+1} a_{n+i+2} \cdots a_{n+k}}{a_{n+1} a_{n+2} \cdots a_{n+k-i}}\right|<\infty .
$$

(For brevity denote the expression inside the above summations by $a_{n k i}$.)

(b) The basis is an $N$-basis with $\left\|T_{1}\right\|=1$ and for each $n \geqq 0$, there is an $m>0$ such that $\sum_{j=m}^{\infty} \sup _{i \geqq m} a_{n(i+j) i}<\infty$.

(c) The basis is the usual basis of the one-sided $c_{0}$ space, $I^{+}=0$, and for each $n \geqq 0, \sup _{i \geq 0} \sum_{j=0}^{i} a_{n i j}<\infty$.

(d) The basis is the usual one in the one-sided $l_{1}$ space, for each $n \geqq 0$, $\sup _{i, j \geq 0} a_{n(i+j) i}<\infty$, and $I^{+}=0$.

Proof. The conditions in (a), (b), (c) and (d) guarantee that $I^{+}=0$ and that $\boldsymbol{B}_{n}$ is an algebra for all $n \geqq 0$. Theorems $22,23,21$ and 21 again are used in the respective four parts. (Although Theorem 21 is stated for two-sided bases, the corresponding result for one-sided bases is true and proven similarly.)

Note 3. Using different methods N. K. Nikol'skii [5], [16] has discovered very similar results to (b), (c) and (d).

The condition: $\left|a_{n}\right|$ decreases monotonically and $\left\{a_{n}\right\}_{1}^{\infty} \in l_{p}$ for some $p \geqq 1$, is stronger than all four conditions of the above corollary.

In [15], Donoghue gave the first example of a weighted shift whose only invariant subspaces are of the form $\boldsymbol{B}_{n}$. His example: $\left\{y_{n}\right\}_{0}^{\infty}$ an orthonormal basis in Hilbert space, $a_{n}=2^{-n}$.

Nikol'skir [12] has found shifts in $l_{p}$ for which $a_{n} \neq 0$ (all $n$ ), and $a_{n} \rightarrow 0$ but which have additional invariant subspaces of a different form.

A shift on the usual basis of $l_{1}$ where the sequence of "weights" $a=1,1 / 2,1,1 / 2$, $1 / 4,1 / 2,1,1 / 2,1 / 4,1 / 8,1 / 4, \ldots$ satisfies condition (d) of the corollary. This gives an example of an operator $T$ no polynomial of which is compact, and whose lattice of invariant subspaces is order anti-isomorphic to the positive integers.

Some development of the theory of the adjoint of $T$ is necessary in order to prove the next theorem.

To each bounded linear functional $F$ on $B$ we shall assign the "Laurent series" $\sum b_{n} z^{n}$, where $b_{n}=\left(z^{-n}, F\right)=F\left(z^{-n}\right)$. We say $f(z)=\sum b_{n} z^{n} \in B^{*}$ with norm $\|f(z)\|^{*}$ $=\|F\|$. If $f(z) \in B^{*}$ so is $T f(z)=z f(z)$ and we have $(T g(z), f(z))=(g(z), T f(z))$ for all $g \in \boldsymbol{B}$.

For $g(z)=\sum c_{n} z^{n} \in \boldsymbol{B}$ we have $\left|\left(g, z^{n}\right)\right|=\left|c_{-n}\right| \leqq M\|g\| /\left\|z^{-n}\right\|$, (using (2.1)). Thus $\left\|z^{n}\right\|^{*} \leqq M /\left\|z^{-n}\right\|$. But $1=\left(z^{-n}, z^{n}\right) \leqq\left\|z^{-n}\right\|\left\|z^{n}\right\|^{*}$. Thus $\left\|z^{n}\right\|^{*} \geqq 1 /\left\|z^{-n}\right\|$. Finally note that for $f(z)=\sum b_{n} z^{n} \in B^{*},\left|b_{n}\right|=\left|\left(z^{-n}, f(z)\right)\right| \leqq\|f(z)\|^{*}\left\|z^{-n}\right\| \leqq M\|f(z)\|^{*} /\left\|z^{n}\right\|^{*}$, which is the analogue for $B^{*}$ of $(2.1)$. 
It follows that the same proofs and statements as those in Theorems 11 and 12 hold for $B^{*}$ with

$$
\begin{array}{cl}
I^{+*}=Q^{-}, & I^{-*}=Q^{+}, \quad Q^{+*}=I^{-}, \quad Q^{-*}=I^{+}, \\
R_{1}^{*}=R_{1}, & \text { and } R_{2}^{*}=R_{2} .
\end{array}
$$

(Be it understood, however, that $\left\{z^{n}\right\}_{-\infty}^{\infty}$ may be no Schauder basis in $B^{*}$.)

THEOREM 25. Suppose $f^{+}$is analytic on a neighborhood of the set $|z| \leqq Q^{+}$and $f^{-}$is analytic on a neighborhood of $|z| \geqq Q^{-}$. Then $E_{f}$ is either of finite dimension, finite codimension, or is $\boldsymbol{B}$ itself.

In particular, if $I^{-} \leqq I^{+}$, then $E_{f}$ is the set of all series in $B$ "vanishing as $f$ does". We know necessary and sufficient conditions that $E_{f}$ is finite dimensional (\$9). In all other cases $\boldsymbol{E}_{f}=\boldsymbol{B}$.

Proof. Case 1. Suppose $Q^{-} \leqq Q^{+}$. (This includes the case where $I^{-} \leqq I^{+}$.) Choose real positive $s$ and $t$ such that $s<Q^{-}, Q^{+}<t, f(z)$ is analytic on a neighborhood of the annulus $s \leqq|z| \leqq t$, and $f(z)$ has no zero on the zero-centered circles of radii $s$ and $t$.

Let $\left\{y_{n}^{\prime}\right\}_{-\infty}^{\infty}$ be the usual basis of $l_{1}=\boldsymbol{B}^{\prime}$ and let $\boldsymbol{a}^{\prime}=\left\{a_{n}^{\prime}\right\}_{-\infty}^{\infty}$ be defined by $a_{n}=t$ for $n \geqq 0$ and $a_{n}=s$ for $n<0$. These conditions define a shift operator $T$ and a power series structure on $\boldsymbol{B}^{\prime}$. An easy calculation using Theorem 21 shows that $\boldsymbol{B}^{\prime}$ is an algebra. We have $R_{1}^{\prime}=Q^{\prime+}=I^{\prime+}=t$ and $R_{2}^{\prime}=Q^{\prime-}=I^{\prime-}=s$.

If $g(z) \in B^{\prime}, g^{+}(z)$ is analytic in the region $|z|<t$ (Theorem 11). But $t>Q^{+}$, so by Theorem $12, g^{+}(z) \in B$. Similarly $g^{-} \in B$, so $g \in B$. The map from $B^{\prime}$ to $B$ which sends the series $g(z)$ to the series $g(z)$, being defined by a matrix (the infinite unit matrix with respect to the bases $\left\{z^{n}\right\}_{-\infty}^{\infty}$ in $B^{\prime}$ and $B$ ), is bounded. We conclude that convergence in $\boldsymbol{B}^{\prime}$ implies convergence in $\boldsymbol{B}$.

$f^{+}(z)$ is analytic on a neighborhood of the region $|z| \leqq Q^{+}=t$ so $f^{+}(z) \in \boldsymbol{B}^{\prime}$. Similarly $f^{-}(z) \in \boldsymbol{B}^{\prime}$ so $f(z) \in \boldsymbol{B}^{\prime}$. Since $f(z)$ has no zeros on the circles of radii $R_{1}^{\prime}$ and $R_{2}^{\prime}$, Theorem 18 shows that the smallest doubly invariant subspace of $B^{\prime}$ containing $f(z)$ is finite codimensional. This subspace is spanned by the elements $\left\{z^{n} f(z)\right\}_{-\infty}^{\infty}$. Thus this sequence holds a polynomial in its $\boldsymbol{B}^{\prime}$ span. It follows that it holds a polynomial $p$ of degree $m$ in its $B$ span, which lies within $E_{f}$. As in Lemma 1, we see that $\left[z^{n}\right]_{0}^{m-1}$ spans $\boldsymbol{M}=\boldsymbol{B} / \boldsymbol{E}_{f} . \boldsymbol{E}_{f}$ is finite codimensional.

Finally as in the end of the proof of Theorem 18, we can conclude that $E_{f}$ must be the set of elements in $B$ "vanishing as $f$ does".

Case 2. Suppose $Q^{+}<Q^{-}$. The positive and negative parts of each of the series $\left\{z^{n} f\right\}_{-\infty}^{\infty}$ are analytic respectively on a neighborhood of $|z| \leqq Q^{+}$and of $Q^{-} \geqq|z|$. Thus these series lie in $B$ and in particular in $E_{f}$. If we suppose $E_{f} \neq B$, then there is a series $g(z) \in B^{*}$ such that $\left(z^{n} f, g\right)=0$ for all $n$. By (13.1), the series $g(z)$ converges on the annulus $Q^{+}<|z|<Q^{-}$.

Pick positive real $s$ and $t$ with $Q^{+}<s<t<Q^{-}$, such that $f^{+}(z)$ is analytic on a neighborhood of $|z| \leqq s, f^{-}(z)$ is analytic on a neighborhood of $t \leqq|z|$ and such that $g(z)$ has no zeros on the zero-centered circles of radii $s$ and $t$. 
Let $\boldsymbol{B}^{\prime}$ be the same space constructed in Case 1, using the above $s$ and $t$. Since $Q^{\prime-}=s$ and $Q^{\prime+}=t$, we know that $g(z) \in \boldsymbol{B}^{\prime}$. Since the operator $T^{-1}$ is bounded in $\boldsymbol{B}^{\prime}$ we see that the series $\left\{z^{n} g\right\}_{-\infty}^{\infty}$ all lie in $\boldsymbol{B}^{\prime}$ and that their span is $\boldsymbol{E}_{\boldsymbol{g}}^{\prime}$, the smallest doubly invariant subspace of $\boldsymbol{B}^{\prime}$ containing $g$. Since $\boldsymbol{B}^{\prime}$ is an algebra, Theorem 18 shows that $\boldsymbol{E}_{g}^{\prime}$ has finite codimension in $\boldsymbol{B}^{\prime}$.

By (13.1), for $\boldsymbol{B}^{\prime *}$, the dual of $\boldsymbol{B}^{\prime}$ we have $Q^{\prime-*}=t$ and $Q^{\prime+*}=s$ and so $z^{n} f(z) \in B^{\prime *}$ for all $n$. Now for all $n$ and $m,\left(z^{m} g, z^{n} f\right)=\left(g, z^{n+m} f\right)=0$. Thus $\left\{z^{n} f \mid-\infty<n<\infty\right\}$ lies in the annihilator of $\boldsymbol{E}_{g}^{\prime}$. But the annihilator of a finite codimensional subspace is finite dimensional. Hence $\boldsymbol{E}_{f}$ is finite dimensional.

NoTE 4. This theorem has two generalizations to one-sided shifts. The first says that if the radius of convergence of a nonzero $f(z)$ is greater than $Q^{+}$, then $C_{f}$ is finite codimensional. To prove it use a Case 1 type proof as in the theorem and apply the note after Theorem 18.

The other generalization concerns left shifts. A left shift may be defined as the linear map which acts on a basis $\left\{y_{n}\right\}_{0}^{\infty}$ by sending $y_{0}$ to 0 and $y_{n}$ to $\left(a_{n}^{\prime}\right)^{-1} y_{n-1}$ $(n>0)$ for some scalar sequence $\left\{a_{n}^{\prime}\right\}_{1}^{\infty}$. Using the same power series representation as we have been, the left shift sends $f(z)$ to $\left(f(z)-(f)_{0} 1\right) / z$. Note that for a two-sided shift the mapping $T_{-}: f^{-}(z) \rightarrow\left(T f^{-}(z)\right)^{-}$is similar to a left shift on a one-sided basis (with $a_{n}^{\prime}=\left(a_{-n}\right)^{-1}$ ). If $f^{-}(z)$ is analytic on a neighborhood of $|z| \geqq Q^{-}$, and is

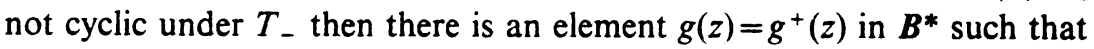

$$
\left(\left(T_{-}\right)^{n} f^{-}, T^{m} g^{+}\right)=0
$$

for all $n, m \geqq 0$. A proof similar to Case 2 shows $f^{-}$generates a finite dimensional subspace under the action of $T_{\text {-. }}$. Translating to left shifts we obtain: If the power series $f(z)$ is analytic on a neighborhood of $|z| \leqq Q^{+}$, then either $f(z)$ is cyclic under the left shift or generates a finite dimensional left invariant subspace. Simple considerations (as in $\S 9$ ) show this second possibility can occur iff $f(z)$ is a rational function.

This property of left shifts was proven for $\left\{y_{n}\right\}_{0}^{\infty}$ an orthonomal basis in Hilbert space and $a_{n}^{\prime}=1$, for all $n$, in [6]. The proof of Theorem 25, Case 2, is a modification of the proof of Theorem 3 in [7].

14. Further analysis of the spectrum. In Theorems 14 and 15 we have found the point spectrum of $T$ and the set of $\lambda$ such that $\mathscr{C} \ell((\lambda-T) B) \neq B$. It follows from these theorems that these sets do not intersect since $\mathscr{E}_{\lambda}$ is not defined at $\sum_{-\infty}^{\infty} \lambda^{-n} z^{n}$. For $\lambda$ in the remainder of the spectrum $\lambda-T$ is $1-1$ and $(\lambda-T) B$ is dense in $B$. What is the range of $\lambda-T$ ?

THEOREM 26. (1) If $Q^{+}<|\lambda|$ or if $|\lambda|<Q^{-}$then the range of $\lambda-T$ includes all $f(z) \in B$ such that $f^{+}(z)$ is analytic on a neighborhood of $|z| \leqq Q^{+}$and $f^{-}(z)$ is analytic on a neighborhood of $Q^{-} \leqq|z|$, e.g. $f=y_{n}$. But (2) if $Q^{-}<|\lambda|<Q^{+}, f(z)$ is in the range of $\lambda-T$, and the series $f(z)$ converges in a neighborhood of $\lambda$, then $f(\lambda)=0$, e.g. $f \neq y_{n}$. 
Proof. (1) Suppose $Q^{+}<|\lambda|$. The proof for $|\lambda|<Q^{-}$is similar. Suppose $f(z)$ is as described in the hypothesis.

Case 1. In addition $|\lambda|<Q^{-}$. Then $(\lambda-z)_{+}^{-1} f^{+}(z)$ is analytic on a neighborhood of $|z| \leqq Q^{+},(\lambda-z)_{-}^{-1} f^{-}(z)$ is analytic on a neighborhood of $Q^{-} \leqq|z|$, so these series lie in $B$. Direct calculation shows $(\lambda-T)$ applied to the sum of these series yields $f(z)$.

Case 2. In addition $Q^{-} \leqq|\lambda|$. Again $(\lambda-z)_{+}^{-1} f^{+}(z)$ lies in $B$. The product $(\lambda-z)_{+}^{-1} f^{-}(z)$ converges to an analytic function on $r<|z|<|\lambda|$, where $r<Q^{-}$, so this series lies in $B$, too. Again, $(\lambda-T)$ applied to the sum of these series yields $f(z)$.

(2) Let $(\lambda-T) g(z)=f(z)$ for some $g(z) \in B$. It is sufficient to show that $g(z)$ is analytic in a neighborhood of $\lambda$. We will prove $g^{+}(z)$ is analytic there. $g^{-}(z)$ can be shown analytic there in a similar manner.

$(\lambda-z) g^{+}(z)=f^{+}(z)+$ constant. Thus if $g^{+}(z)$ is not analytic in a neighborhood of $\lambda$, the radius of convergence of $g^{+}(z)$ must be $|\lambda|$, and $g^{+}(z)$ must have a single pole at $\lambda$. Theorem 13 is concerned with power series whose radius of convergence is less than $Q^{+}$. It shows that $g^{+}(z)=g_{1}(z)+g_{2}(z)$ where $g_{2}(z)$ is analytic in a neighborhood of $\lambda$, while $g_{1}(z)$ has Hadamard gaps in its power series and must have the same singularity on its circle of convergence as $g^{+}(z)$. But a series with Hadamard gaps cannot have an isolated pole on its circle of convergence [8, Chapter XI].

We shall outline a description of the sets

$$
S_{1}=\{\lambda \mid \operatorname{kernel}(\lambda-T) \neq 0 \text { and }(\lambda-T) B=B\}
$$

and

$$
S_{2}=\{\lambda \mid(\lambda-T) B \text { is closed but not equal to } B\} .
$$

We know for $\lambda \in S_{1}, \operatorname{dim}(\operatorname{ker}(\lambda-T))=1, Q^{+} \leqq|\lambda| \leqq Q^{-}$and $\lambda \neq 0$. For $\lambda \in S_{2}$, $\operatorname{codim}(\lambda-T) B=1, I^{-} \leqq|\lambda| \leqq I^{+}$and $\lambda \neq 0$. By [9, pp. 369-370], $\lambda \in S_{1}$ iff $(\lambda-T)$ has a right inverse $(\lambda-T)^{R}$ but no inverse. Also, $\lambda \in S_{2}$ iff $(\lambda-T)$ has a left inverse $(\lambda-T)^{L}$ but no inverse. By [9, Theorem 4, p. 376], $S_{1}$ and $S_{2}$ are open sets.

Suppose $\lambda \in S_{1}$. $(\lambda-T)^{R}$ is not unique. But by easy calculation we may verify $(\lambda-T)^{R} f(z)=(\lambda-z)_{+}^{-1} f^{+}(z)+(\lambda-z)_{-1}^{-1} f^{-}(z)+c e(z)$, where $c$ is some nonfixed scalar and $e(z)=\sum_{-\infty}^{\infty} \lambda^{-n} z^{n}$. But $e(z) \in B$. We may conclude that $\lambda \in S_{1}$ iff the mappings

$$
f^{+}(z) \rightarrow(\lambda-z)_{+}^{-1} f^{+}(z)
$$

and

$$
\left.f^{-}(z) \rightarrow(\lambda-z)\right)^{-1} f^{-}(z)
$$

are bounded (since these mappings are defined by matrices). We may now let $c=0$ above to fix $(\lambda-T)^{R}$.

Suppose $\lambda \in S_{2} .(\lambda-T)^{L}$ is not unique. But we can fix it by choosing a subspace $\boldsymbol{H}_{\mathbf{2}}$ satisfying $(\lambda-T) B \oplus \boldsymbol{H}_{2}=\boldsymbol{B}$ and letting kernel $(\lambda-T)^{L}=\boldsymbol{H}_{2}$ (see [10, Theorem 
1, p. 209] for details). We shall choose $H_{2}=$ the constant functions. Then by easy calculation we may verify $(\lambda-T)^{L} f(z)=\left((\lambda-z)_{-1}^{-1} f^{+}(z)\right)^{+}+\left((\lambda-z)_{+}^{-1} f^{-}(z)\right)^{-}$. We conclude that $\lambda \in S_{2}$ iff the mappings

$$
\left.f^{+}(z) \rightarrow((\lambda-z))^{-1} f^{+}(z)\right)^{+}
$$

and

$$
f^{-}(z) \rightarrow\left((\lambda-z)_{+}^{-1} f^{-}(z)\right)^{-}
$$

are bounded.

The complement of $S_{1}$ (resp. of $S_{2}$ ) has 1 or 2 components, each component containing the set $Q^{-} \leqq|z|$ or the set $|z| \leqq Q^{+}$(resp. $I^{+} \geqq|z|$ or $\left.|z| \geqq I^{-}\right)$.

Proof. If $\sigma$ were a nonempty compact and relatively open subset of the complement of $S_{1}$, lying in the region $Q^{+}<|z|<Q^{-}$, and $C$ were a properly chosen curve (see [10, p. 218ff.] for details) then $P_{\sigma}=\int_{C}(\lambda-T)^{R} d \lambda$ would be a nontrivial projection. Since $C$ may be chosen not to wind around 0 , then as in Theorem 8 we can easily show $P_{\sigma}=0$. The proof for $S_{2}$ is similar.

Let

$$
\begin{aligned}
& R_{1}^{+}=\lim _{n \rightarrow \infty}\left(\sup _{m \geq 0} \prod_{i=m+1}^{m+n}\left|a_{i}\right|\right)^{1 / n}, \quad R_{2}^{+}=\lim _{n \rightarrow \infty}\left(\inf _{m \geq 0}\right)^{1 / n}, \\
& R_{1}^{-}=\lim _{n \rightarrow \infty}\left(\sup _{m+n \leq 0}\right)^{1 / n}, \text { and } R_{2}^{-}=\lim _{n \rightarrow \infty}\left(\inf _{m+n \leq 0}\right)^{1 / n} .
\end{aligned}
$$

We have $R_{2}^{+} \leqq I^{+} \leqq Q^{+} \leqq R_{1}^{+}$and $R_{2}^{-} \leqq Q^{-} \leqq I^{-} \leqq R_{1}^{-}$. A proof similar to that of Theorem 4 shows mappings (1), (2), (3) and (4) are unbounded if, respectively, $|\lambda|<R_{1}^{+}, \quad|\lambda|>R_{2}^{-}, \quad|\lambda|>R_{2}^{+}, \quad|\lambda|<R_{1}^{-}$. Thus $S_{1} \subseteq\left\{R_{1}^{+}<|z|<R_{2}^{-}\right\}$and $S_{2} \subseteq\left\{R_{1}^{-}<|z|<R_{2}^{+}\right\}$. Equality holds if $\left\{y_{n}\right\}_{-\infty}^{\infty}$ satisfies the hypotheses of Theorem 10. We indicate the method of proof: Let $T_{+}^{-1}$ be the mapping (on the "Taylor series" elements of $\boldsymbol{B}$ ) which sends $\sum_{0}^{\infty} b_{n} z^{n}$ to $\sum_{0}^{\infty} b_{n+1} z^{n}$. As in Theorem 10 we can calculate the spectral radius of $T_{+}^{-1}$ which turns out to be $\left(R_{2}^{+}\right)^{-1}$. Then mapping (3) is $T_{+}^{-1}\left(1-\lambda T_{+}^{-1}\right)^{-1}$.

Finally we consider the possibility of weakening the requirement that $\left\{y_{n}\right\}$ be a Schauder basis. Let us assume $\left\{y_{n}\right\}$ and $\left\{F_{n}\right\}$ are biorthogonal sequences, with $\left\|y_{n}\right\|$ and $\left\|F_{n}\right\|$ uniformly bounded, $\left\{y_{n}\right\}$ complete, and $\left\{F_{n}\right\}$ total.

If $T_{a}$ is defined by the equations $F_{n}\left(T_{a} f\right)=F_{n-1}\left(a_{n} f\right)$, then everything in this paper and [11] remains true (aside from minor modifications in $\$ 10$ ) when $\left\{y_{n}\right\}$ is one-sided. The difficulty lies in Theorem 2, for which a proof is given in [17] in the one-sided case. For the two-sided case everything not depending on Theorem 2 remains true, i.e. \$\$7-9 and 11-14. Moreover, using alternate but similar proofs Theorems $1,3,4,6,7,8$ and 10 remain true if $f(T)$ is redefined to be the operator satisfying $f(T) 1=f(z)$.

\section{REFERENCES}

1. E. R. Lorch, The theory of analytic functions in normed Abelian vector rings, Trans. Amer Math. Soc. 54 (1943), 414-425.

2. A. E. Taylor, Functional analysis, Wiley, New York, 1958. 
3. H. Helson, Lectures on invariant subspaces, Academic Press, New York, 1964.

4. P. R. Halmos, Review: Lectures on invariant subspaces (Henry Helson), Bull. Amer. Math. Soc. 71 (1965), 490-494.

5. N. K. Nikol'skiĭ, Invariant subspaces of certain completely continuous operators, Vestnik Leningrad. Univ. 20 (1965), 68-77.

6. R. G. Douglas, H. S. Shapiro and A. L. Shields, On cyclic vectors of the backward shift, Bull. Amer. Math. Soc. 73 (1967), 156-159.

7. H. S. Shapiro, Weakly invertible elements in certain function spaces, and generators in $l_{1}$, Michigan Math. J. 11 (1964), 161-165.

8. P. Dienes, The Taylor series, Clarendon Press, Oxford, 1931.

9. P. Saphar, Contributions à l'étude des applications linéaire dans un espace de Banach, Bull. Soc. Math. France 92 (1964), 363-384.

10. - Sur les applications linéaires dans un espace de Banach. II, Ann. Sci. École Norm. Sup. (3) 82 (1965), 205-240.

11. R. Gellar, Operators commuting with a weighted shift, Proc. Amer. Math. Soc. (to appear).

12. N. K. Nikol'skiï, Invariant subspaces of weighted shift operators, Mat. Sb. 74(116) (1967), 171-190 = Math. USSR Sb. 3 (1967), 159-176.

13. P. R. Halmos, A Hilbert space problem book, Van Nostrand, New York, 1967.

14. P. A. Fuhrmann, On the corona theorem and its application to spectral problems in Hilbert space, Trans. Amer. Math. Soc. 132 (1968), 55-66.

15. W. F. Donoghue, The lattice of invariant subspaces of a completely continuous quasinilpotent transformation, Pacific J. Math. 7 (1957), 1031-1035.

16. N. K. Nikol'skii, Basisness and unicellularity of weighted shift operators, Funkcional. Anal. i Priložen. 2 (1968), 95-96. (Russian)

17. R. Gellar, Shift operators in Banach space, Dissertation, Columbia Univ., New York, 1968.

18. N. K. Nikol'skiir, The unicellularity and nonunicellularity of weighted shift operators, Dokl. Akad. Nauk SSSR 172 (1967), 287-290=Soviet Math. Dokl. 8 (1967), 91-94.

19. - Basisness and unicellularity of weighted shift operators, Izv. Akad. Nauk SSSR Ser. Mat. 32 (1968), 1123-1137 = Math. USSR Izv. 2 (1968), (to appear).

20. - Invariant subspaces and bases of generalized primitives in the sense of $A . O$. Gel'fand-A. F. Leontov, Mat. Issled. 3 (1968), 101-116. (Russian)

21. R. Kelley, Weighted shifts on Hilbert space, Dissertation, University of Michigan, 1966.

University of New MeXico, Albuquerque, New Mexico 